\title{
Parton-shower Effects in Vector-Boson-Fusion Processes
}

\section{Michael Rauch*}

Institute for Theoretical Physics, Karlsruhe Institute of Technology

E-mail: michael.rauch@kit.edu

\section{Simon Plätzer}

Institute for Particle Physics Phenomenology, Durham University

School of Physics and Astronomy, University of Manchester

\begin{abstract}
We investigate the effects of combining next-to-leading order QCD results with parton-shower effects in $W^{+} W^{-} j j$ production via vector-boson fusion including leptonic decays. Using the Herwig 7 framework interfaced to VBFNLO 3, we compare the predictions obtained from the angular-ordered and dipole-based parton shower algorithms combined with subtractive, MC@NLO-type, and multiplicative, Powheg-type, matching. A consistent treatment of renormalisation and factorisation scale variations in the hard process and the parton shower allows to assign more reliable theory uncertainty predictions to key distributions like the central rapidity gap.
\end{abstract}

XXIV International Workshop on Deep-Inelastic Scattering and Related Subjects

11-15 April, 2016

DESY Hamburg, Germany

\footnotetext{
* Speaker.
} 


\section{Introduction}

Production of electroweak bosons via vector-boson fusion and vector-boson scattering, collectively referred to as VBF in the following, is one of the main process classes to study during the run-2 phase of the LHC. It proceeds via the space-like exchange of electroweak bosons between two scattering (anti-)quarks. The characteristic feature of VBF processes are two energetic jets in the forward regions of the detector, the so-called tagging jets [1]. In the central region between the two tagging jets, the jet activity is reduced, which can be exploited for example by a mini-jet veto [2]. This distinguishes them from two classes of background processes which form an irreducible background. These are multi-boson production, where one boson decays into a quark-anti-quark pair, and QCD-induced production in association with two jets. The application of tight VBF cuts, typically an invariant mass of the tagging jets above several hundreds of $\mathrm{GeV}$ and a large rapidity separation between them, reduces the contribution of these background processes and strongly suppresses interference terms with the VBF process [3]. Also, interference effects between $t$ - and $u$-channel boson exchange become negligible and justify using the VBF approximation, where these terms are removed. VBF processes are an ideal tool to study the gauge structure of the SM due to the appearance of triple and, in particular, quartic gauge couplings. Diagrams with quartic and triple gauge vertices as well as with the exchange of a Higgs boson exhibit a strong cancellation among them, which would be spoiled if any of them receives anomalous contributions. Hence, VBF is a sensitive probe to test such effects.

Next-to-leading order (NLO) QCD corrections to all VBF processes have been calculated [4]. Their effect is rather modest, with corrections typically up to $10 \%$. Taking the momentum transfer through the exchanged bosons as a dynamical scale proves to be an advantageous choice. All other higher-order corrections have been established for VBF-Higgs production only so far. The NLO electroweak corrections [5] have a similar size as the NLO QCD ones. NNLO QCD [6] and NNNLO QCD [7] corrections to the inclusive cross section in the structure-function approach are below the percent level and at the 1-2 permill level, respectively. NNLO QCD corrections to differential distributions in the VBF approximation show much larger effects [8] up to 10\%, though at least for some distributions are modelled reasonably well by parton-shower effects matched to NLO QCD calculations. Studies of matching the NLO QCD calculations to parton showers have been performed for some of the VBF processes $[9,10]$ in the POWHEG-BOX framework [11, 12], and for $W^{+} W^{-} j j$ production [13] using Herwig 7 [14] and VBFNLO 3 [15]. To be able to assess the effects, it is necessary to not only compare the central predictions, but also to study the associated theory uncertainties. Their size can be estimated from a variation of the different scales entering the predictions, but also using different matching schemes and parton-shower algorithms. We will thereby focus on the perturbative part of the simulation and not consider any hadronisation or multi-parton-interaction effects.

\section{Calculational Setup}

We perform our study [13] using the Herwig 7 Monte Carlo event generator [14], which is based on HERWIG++ [16]. Two different matching schemes are implemented, namely subtractive, MC@NLO-type matching [17], and multiplicative, Powheg-type matching [11]. These can be 
combined with both parton shower modules available in Herwig 7, the dipole shower [18] and the angular-ordered shower [19]. Its Matchbox module, based on Ref. [20], performs the simulation of NLO QCD events. The necessary squared amplitudes, both for the Born and the real-emission process as well as the Born-virtual interference term, are in our study provided by VBFNLO 3 [15], which allows us to get fast and accurate predictions. The communication between the two programs is done using the BLHA 2 standard [21]. We have extended the interface by the possibility to use the dedicated phase-space generator of VBFNLO 3, and use this for the results shown in the following. As example VBF process we take the electroweak production of $p p \rightarrow W^{+} W^{-} j j \rightarrow$ $e^{+} v_{e} \mu^{-} \bar{v}_{\mu} j j$. The leptonic decays of the $W$ bosons as well as off-shell, non-resonant and Higgs exchange contributions are fully included. For the partons we employ the VBF approximation and neglect interference terms between quarks of the same flavour in the final state. We perform our study for the LHC at a centre-of-mass energy of $13 \mathrm{TeV}$. As we are not interested in top-quark effects, we apply a perfect bottom-quark veto on the final state. At the analysis level we apply typical VBF selection cuts,

$$
\begin{aligned}
& p_{T, j}>30 \mathrm{GeV}, \quad\left|y_{j}\right|<4.5, \quad m_{j 1, j 2}>600 \mathrm{GeV}, \quad\left|y_{j 1}-y_{j 2}\right|>3.6, \\
& p_{T, \ell}>20 \mathrm{GeV}, \quad\left|y_{\ell}\right|<2.5, \quad m_{e^{+}, \mu^{-}}>15 \mathrm{GeV} .
\end{aligned}
$$

Jets are clustered from massless partons via the anti- $k_{T}$ algorithm [22] with a cone radius of $R=0.4$. As PDF set MMHT2014 [23] is used.

\section{Results}
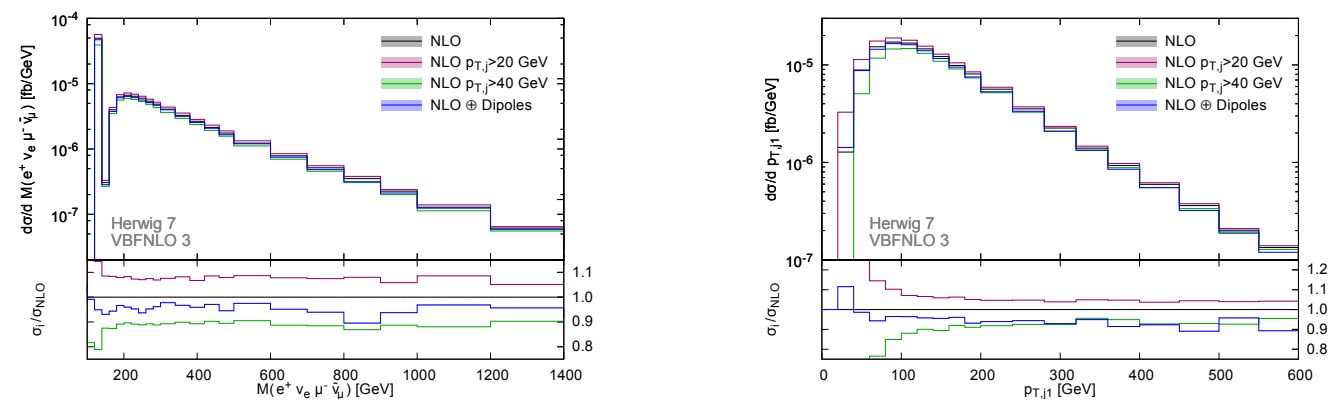

Figure 1: Invariant mass of the four-lepton system (left) and transverse momentum of the leading jet (right). Shown is the fixed-order NLO prediction applying different cuts on the jet transverse momentum and matched with the dipole shower. Right panel taken from Ref. [13].

Parton-shower emissions can change the kinematic properties of an event, if the emission is sufficiently hard and emitted under a wide angle such that it is not clustered back into the emitting jet. This in turn can lead to a migration across cut boundaries, typically reducing the cross section. How this affects our process is shown in Fig. 1. Here we show two differential distributions, namely the four-lepton invariant mass and the transverse momentum of the leading jet. In both cases, the parton-shower calculation leads to consistently lower cross sections than the fixed-order result. For comparison, we also show fixed-order results where the transverse momentum cut has been lowered to $20 \mathrm{GeV}$, the generation-level cut, or increased to $40 \mathrm{GeV}$, which is expected to mimic 

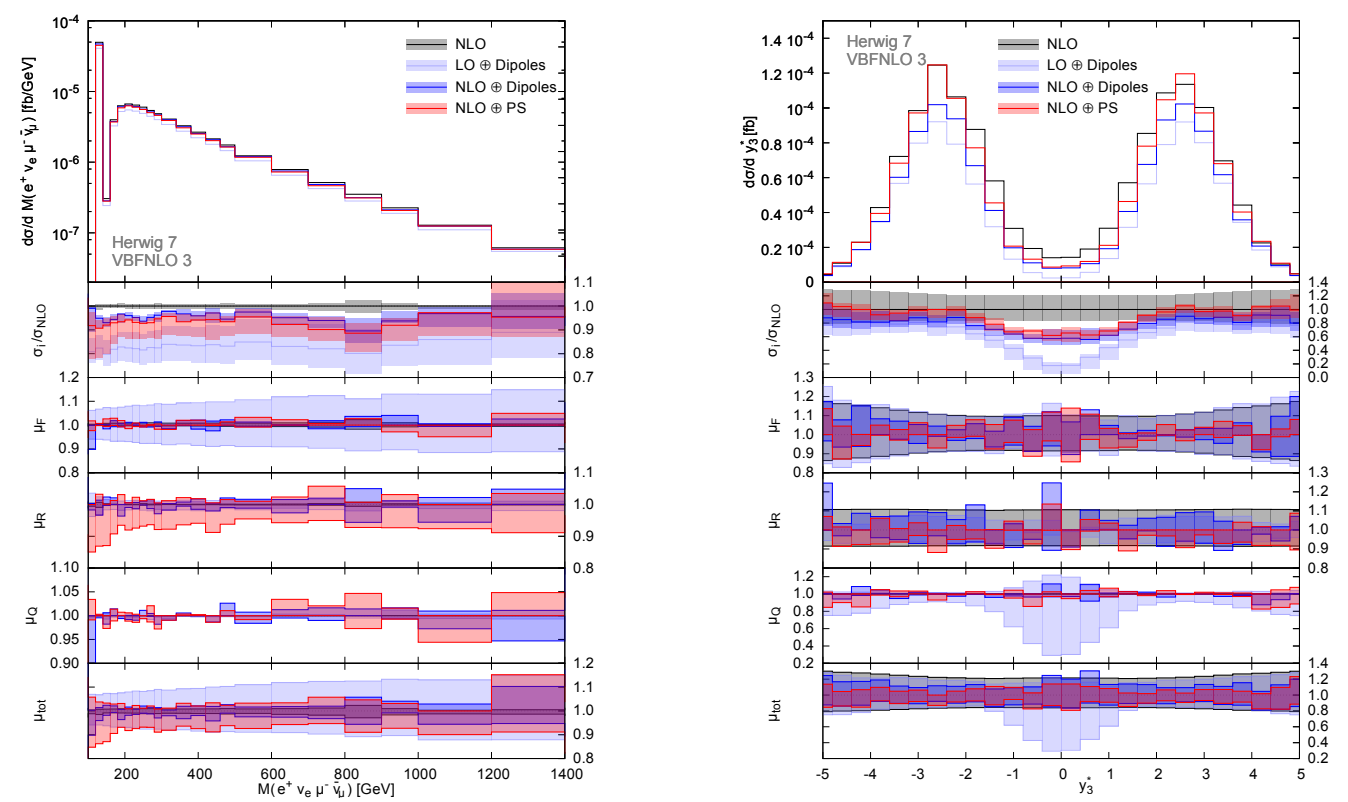

Figure 2: Invariant mass of the four-lepton system (left) and rapidity of the third jet relative to the two tagging jets (right), comparing parton-level NLO results (black), LO plus dipole shower (light blue), and NLO results combined with the dipole (dark blue) and angular-ordered (red) shower using the subtractive matching procedure. Each panel shows, from top to bottom, the central prediction, central prediction and overall scale variation normalised to the fixed-order result, and variation bands for factorisation $\left(\mu_{F}\right)$, renormalisation $\left(\mu_{R}\right)$, and hard veto $\left(\mu_{Q}\right)$ scale as well as the overall envelope $\left(\mu_{\text {tot }}\right)$ normalised to the respective central prediction. Figure taken from Ref. [13].
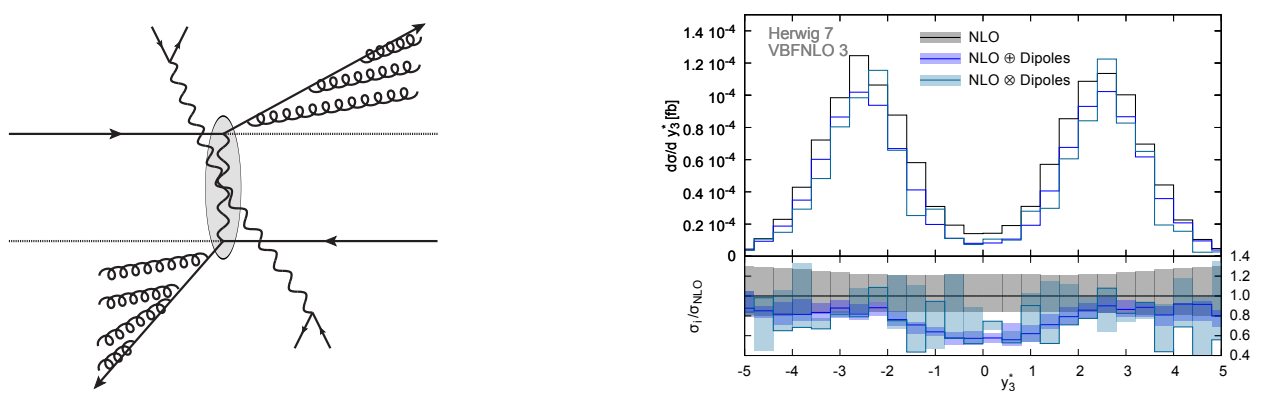

Figure 3: Left: Schematic picture of additional radiation generated by the parton shower. Right: Rapidity of the third jet relative to the two tagging jets, comparing the fixed-order NLO result and NLO combined with the dipole shower using both subtractive and multiplicative matching. Left panel taken from Ref. [13].

parton-shower effects in size. We find that the reduction of the cross section is mostly due to events where additional radiation leads to a reduction of the invariant mass of the tagging jets, thus more events fail this VBF cut.

Turning to uncertainties and parton-shower matching systematics, we present in Fig. 2 in the left panel the invariant-mass distribution of the four leptons for calculations of fixed-order NLO, LO plus dipole shower, as well as NLO combined with dipole and angular-ordered shower using subtractive matching. The lower panels show the cross sections normalised to the fixed-order result, 
and variation bands for factorisation $\left(\mu_{F}\right)$, renormalisation $\left(\mu_{R}\right)$, and hard veto $\left(\mu_{Q}\right)$ scale as well as the overall envelope $\left(\mu_{\mathrm{tot}}\right)$ normalised to the respective central prediction. Individual scales are varied by a factor of $2^{ \pm 1}$ around the central value, taken as the transverse momentum of the leading jet. This dynamical scale is directly related to the momentum transfer of the exchanged bosons, and also an important quantity in the parton-shower algorithm, thus making it an ideal choice. For the overall band, we allow the different scales to vary independently, provided the ratio of any two scales is within the range $\left[\frac{1}{2} ; 2\right]$ as well. As expected for a quantity constructed from the electroweak subsystem, shower effects on the shape are small, while the inclusive rate gets reduced due to events migrating outside the allowed cut range. The transition from LO $\oplus$ PS to NLO $\oplus$ PS leads to a clearly reduced scale uncertainty. The increased renormalisation dependence of NLO $\oplus$ PS at small invariant masses is due to a larger value of the strong coupling constant, which leads to more radiation and thus larger migration effects. The right panel of Fig. 2 shows the rapidity of the third jet relative to the two tagging jets, $y_{3}^{*}=y_{3}-\frac{y_{1}+y_{2}}{2}$. This quantity is purely due to shower effects in the LO $\oplus$ PS simulation, and of leading-order accuracy for all other curves. Consequently, we observe much larger effects, both in changes of the shape as well as bigger uncertainty bands. In particular the LO $\oplus$ PS curve exhibits a large dependency on the shower hard scale and also a strongly reduced cross section in the central region. The reason for this becomes clear when considering the schematic picture shown in Fig. 3 on the left. As the colour correlation of the tagging jet is solely with the beam remnant, but not with the other quark line as the exchanged boson is a colour singlet, one expects that additional radiation is generated predominantly between the tagging jet and the beam line, and thus for large absolute values of rapidity. Corrections by the hard matrix element when using the NLO calculation increase this prediction somewhat, but again both parton showers reduce the rate in the central region compared to the fixed-order result. This also holds for the multiplicative matching scheme, which one can see from the comparison shown in the right panel of Fig. 3. For this scheme we use the resummation profile [24], and the bands in the ratio denote a joint variation of all four scales by a factor $2^{ \pm 1}$.

In summary, matching and parton-shower uncertainties are well under control for this process.

\section{Acknowledgments}

We would like to thank the organisers for the stimulating atmosphere at the conference. We are grateful to the other members of the Herwig and VBFNLO collaborations for encouragement and helpful discussions. SP acknowledges support by a FP7 Marie Curie Intra European Fellowship under Grant Agreement PIEF-GA-2013-628739.

\section{References}

[1] D. Zeppenfeld, hep-ph/9902307.

[2] D. L. Rainwater, R. Szalapski and D. Zeppenfeld, Phys. Rev. D 54 (1996) 6680 [hep-ph/9605444].

[3] F. Campanario, M. Kerner, L. D. Ninh and D. Zeppenfeld, Phys. Rev. D 89 (2014) no.5, 054009 [arXiv:1311.6738 [hep-ph]].

[4] T. Figy, C. Oleari and D. Zeppenfeld, Phys. Rev. D 68 (2003) 073005 [hep-ph/0306109]; C. Oleari and D. Zeppenfeld, Phys. Rev. D 69 (2004) 093004 [hep-ph/0310156]; B. Jäger, C. Oleari and 
D. Zeppenfeld, JHEP 0607 (2006) 015 [hep-ph/0603177]; B. Jäger, C. Oleari and D. Zeppenfeld, Phys. Rev. D 73 (2006) 113006 [hep-ph/0604200]; G. Bozzi, B. Jäger, C. Oleari and D. Zeppenfeld, Phys. Rev. D 75 (2007) 073004 [hep-ph/0701105]; B. Jäger, C. Oleari and D. Zeppenfeld, Phys. Rev. D 80 (2009) 034022 [arXiv:0907.0580 [hep-ph]]; A. Denner, L. Hosekova and S. Kallweit, Phys. Rev. D 86 (2012) 114014 [arXiv:1209.2389 [hep-ph]]; F. Campanario, N. Kaiser and D. Zeppenfeld, Phys. Rev. D 89 (2014) no.1, 014009 [arXiv:1309.7259 [hep-ph]]; F. Campanario, T. M. Figy, S. Plätzer and M. Sjödahl, Phys. Rev. Lett. 111 (2013) no.21, 211802 [arXiv:1308.2932 [hep-ph]].

[5] M. Ciccolini, A. Denner and S. Dittmaier, Phys. Rev. Lett. 99 (2007) 161803 [arXiv:0707.0381 [hep-ph]] and Phys. Rev. D 77 (2008) 013002 [arXiv:0710.4749 [hep-ph]]; T. Figy, S. Palmer and G. Weiglein, JHEP 1202 (2012) 105 [arXiv:1012.4789 [hep-ph]].

[6] P. Bolzoni, F. Maltoni, S. O. Moch and M. Zaro, Phys. Rev. Lett. 105 (2010) 011801 [arXiv:1003.4451 [hep-ph]] and Phys. Rev. D 85 (2012) 035002 [arXiv:1109.3717 [hep-ph]].

[7] F. A. Dreyer and A. Karlberg, arXiv:1606.00840 [hep-ph].

[8] M. Cacciari, F. A. Dreyer, A. Karlberg, G. P. Salam and G. Zanderighi, Phys. Rev. Lett. 115 (2015) no.8, 082002 [arXiv:1506.02660 [hep-ph]].

[9] P. Nason and C. Oleari, JHEP 1002 (2010) 037 [arXiv:0911.5299 [hep-ph]]; B. Jäger and G. Zanderighi, JHEP 1111 (2011) 055 [arXiv:1108.0864 [hep-ph]]; B. Jäger, S. Schneider and G. Zanderighi, JHEP 1209 (2012) 083 [arXiv:1207.2626 [hep-ph]]; F. Schissler and D. Zeppenfeld, JHEP 1304 (2013) 057 [arXiv:1302.2884]; B. Jäger, A. Karlberg and G. Zanderighi, JHEP 1403 (2014) 141 [arXiv:1312.3252 [hep-ph]]; B. Jäger, F. Schissler and D. Zeppenfeld, JHEP 1407 (2014) 125 [arXiv:1405.6950 [hep-ph]].

[10] B. Jäger and G. Zanderighi, JHEP 1304 (2013) 024 [arXiv:1301.1695 [hep-ph]].

[11] P. Nason, JHEP 0411 (2004) 040 [hep-ph/0409146].

[12] S. Frixione, P. Nason and C. Oleari, JHEP 0711 (2007) 070 [arXiv:0709.2092 [hep-ph]]; S. Alioli, P. Nason, C. Oleari and E. Re, JHEP 1006 (2010) 043 [arXiv:1002.2581 [hep-ph]].

[13] M. Rauch and S. Plätzer, arXiv:1605.07851 [hep-ph].

[14] J. Bellm et al., Eur. Phys. J. C 76 (2016) no.4, 196 [arXiv:1512.01178 [hep-ph]]; https://herwig.hepforge.org/

[15] K. Arnold et al., Comput. Phys. Commun. 180 (2009) 1661 [arXiv:0811.4559 [hep-ph]]; J. Baglio et al., arXiv:1404.3940 [hep-ph]; https://www.itp.kit.edu/vbfnlo/

[16] M. Bähr et al., Eur. Phys. J. C 58 (2008) 639 [arXiv:0803.0883 [hep-ph]].

[17] S. Frixione and B. R. Webber, JHEP 0206 (2002) 029 [hep-ph/0204244].

[18] S. Plätzer and S. Gieseke, JHEP 1101 (2011) 024 [arXiv:0909.5593 [hep-ph]].

[19] S. Gieseke, P. Stephens and B. Webber, JHEP 0312 (2003) 045 [hep-ph/0310083].

[20] S. Plätzer and S. Gieseke, Eur. Phys. J. C 72 (2012) 2187 [arXiv:1109.6256 [hep-ph]].

[21] S. Alioli et al., Comput. Phys. Commun. 185 (2014) 560 [arXiv:1308.3462 [hep-ph]].

[22] M. Cacciari, G. P. Salam and G. Soyez, JHEP 0804 (2008) 063 [arXiv:0802.1189 [hep-ph]].

[23] L. A. Harland-Lang, A. D. Martin, P. Motylinski and R. S. Thorne, Eur. Phys. J. C 75 (2015) no.5, 204 [arXiv:1412.3989 [hep-ph]].

[24] J. Bellm, G. Nail, S. Plätzer, P. Schichtel and A. Siódmok, arXiv:1605.01338 [hep-ph]. 